\title{
Role of EUS-FNA for gallbladder mass lesions with biliary obstruction: a large single-center experience
}

\section{다 (i)}

\author{
Authors \\ Vikas Singla', Rachit Agarwal' ${ }^{2}$, Shrihari Anil Anikhindi ${ }^{1}$, Pankaj Puri ${ }^{1}$, Mandhir Kumar ${ }^{1}$, Piyush Ranjan ${ }^{1}$, Ashish \\ Kumar $^{1}$, Praveen Sharma ${ }^{1}$, Naresh Bansal ${ }^{1}$, Pooja Bakshi ${ }^{3}$, Kusum Verma ${ }^{3}$, Anil Arora ${ }^{1}$
}

Institutions

1 Institute of Liver, Gastroenterology and Pancreaticobiliary Sciences, Sir Ganga Ram Hospital, New Delhi, India

2 Institute of Gastro Sciences, Apollo Gleneagles Hospital, Kolkata, India

3 Department of Cytopathology, Sir Ganga Ram Hospital, New Delhi, India

submitted 29.1.2019

accepted after revision 8.7.2019

\author{
Bibliography \\ DOI https://doi.org/10.1055/a-0982-2862 | \\ Endoscopy International Open 2019; 07: E1403-E1409 \\ (c) Georg Thieme Verlag KG Stuttgart · New York \\ eISSN 2196-9736
}

\section{Corresponding author}

Dr. Vikas Singla, Consultant and Associate Professor, Institute of Liver, Gastroenterology and Pancreaticobiliary Sciences, Sir Ganga Ram Hospital, New Delhi, India Fax: +91-11-25861002

singlavikas1979@gmail.com

\section{ABSTRACT}

Background and study aims Although endoscopic ultrasound (EUS)-guided fine-needle aspiration (EUS-FNA) is an established modality for pathological sampling of pancreatic and biliary lesions, limited data are available on the diagnostic value of EUS-FNA for evaluation of gallbladder mass lesions, a common cause of obstructive jaundice. We aimed to evaluate the usefulness of EUS-FNA for diagnosis of gallbladder mass lesions presenting with biliary obstruction.

Patients and methods This study was a retrospective analysis of data from patients who had undergone EUS-FNA for gallbladder mass lesions. FNA was performed on either a gallbladder mass, metastatic node or liver lesions. Outcome measures were diagnostic yield of EUS FNA and adverse events.

Results From April 2011 to August 2018, 101 patients with gallbladder mass lesions with biliary obstruction underwent EUS-FNA. The final diagnosis was malignancy in 98, benign disease in one, and two patients were lost to follow-up. EUS-FNA confirmed the diagnosis in 89 of 98 patients with malignancy (sensitivity $90.81 \%$ ); was false negative in nine of 98 cases with malignancy; and was truly negative in the solitary patient with benign disease (specificity $100 \%$ ). Positive predictive value, negative predictive value (NPV), and accuracy were $100 \%, 10 \%$, and $90.90 \%$, respectively. Two patients had self-limiting pain.

Conclusion EUS-FNA is a sensitive tool for evaluation of gallbladder mass lesions presenting with obstructive jaundice. However, because of low NPV, lesions in which FNA is negative should be further evaluated.

\section{Introduction}

Gallbladder cancer is one of the most common abdominal malignancies worldwide and has a high incidence in North India and Chile [1 - 3]. Although such tumors may be diagnosed incidentally on cholecystectomy specimens, the majority of patients are symptomatic and they are more likely to have advanced unresectable disease and poor survival rates [4-6]. Clinical manifestations vary. A patient may be asymptomatic, or may present with abdominal pain, jaundice, weight loss, an- orexia and symptoms of gastric outlet obstruction. One-third of patients develop jaundice, which brings them to the attention of gastroenterologist $[7,8]$, and usually results from obstruction of the bile duct either by tumor arising from the gallbladder neck or metastatic lymph node compression. Jaundice is a marker of poor prognosis, the resection rate is low in these patients $[9,10]$, and surgery is associated with poor outcome [8, $11,12]$.

Treatment options are limited in patients with unresectable disease; chemotherapy does not have significant effect on sur- 
vival [13]. Tissue diagnosis is necessary for potentially untreatable gallbladder cancer and to rule out other treatable conditions such as lymphoma, tuberculosis, and xanthogranulomatous cholecystitis. Endoscopic ultrasound (EUS) has been used for evaluation of proximal biliary obstruction [14-17], but data regarding its utility in gallbladder lesions are limited [1821 ]. All the previous studies had a small sample size and none of the previous studies have specifically studied the role of EUSguided fine needle aspiration (EUS-FNA) in gallbladder lesions presenting with jaundice. The gallbladder can be seen easily from the antrum or duodenal bulb, and FNA can be easily obtained from the mass lesion. We perform EUS for proximal biliary obstruction and FNA is obtained, if a gallbladder mass is seen.

The aim of the current study was to evaluate the role of EUSFNA for pathological diagnosis in a subset of patients with gallbladder mass lesions presenting with biliary obstruction. We chose to selectively analyse the outcome in patients with gallbladder mass lesions and biliary obstruction, as the majority of these lesions are unresectable, and hence tissue diagnosis is necessary. On the other hand, lesions without obstructive jaundice usually arise in the fundus and body, are larger, and either subjected to surgery without tissue diagnosis or are easily accessible with transabdominal ultrasonography.

\section{Patients and methods}

\section{Patients}

At our center, a tertiary care referral center for endoscopic procedures, EUS is routinely performed for patients with proximal biliary obstruction, and FNA is obtained from gallbladder or bile duct mass lesions. From April 2011 to August 2018, data from all patients who underwent EUS for gallbladder mass lesions were prospectively entered in a database. We reviewed the database to collect data on patient demographics, results of EUSFNA, results of follow-up, pathological results of EUS-FNA, results of other modalities for tissue diagnosis (endoscopic retrograde cholangiopancreatography [ERCP] brushings, percutaneous FNA and surgical specimen) and adverse events (AEs). The Institutional Review Board at Sir Ganga Ram Hospital, New Delhi, India approved the study.

Patients with gallbladder lesion and proximal biliary obstruction were included in the study. Proximal biliary obstruction was defined as blockage or compression above the level of insertion of the cystic duct. Gallbladder mass and proximal cholangiocarcinoma can be difficult to differentiate on imaging and patients with mass lesion predominantly involving the gallbladder were considered to have a gallbladder mass. Mass lesions predominantly involving the bile duct were suspected to be cholangiocarcinoma and were excluded. Patients with gallbladder masses without biliary obstruction were also excluded. Patients with biliary obstruction due to concurrent stone disease or metastatic lymph node compression were excluded. Other exclusion criteria included pregnancy, thrombocytopenia $(<50,000 / \mu \mathrm{L})$, prolongation of prothrombin time by more than 4 seconds and intervening large vessel precluding FNA. During EUS examination, a lesion was defined as resectable if there was absence of ascites, involvement of hepatic artery/ portal vein, and liver metastasis, and no enlargement of non-regional nodes. Lesions that would confer a higher stage were targeted before the primary mass. In unresectable lesions, FNA was taken either from the tumor, lymph node or liver lesion, and more than one site was targeted in few patients. In case of resectable lesions, FNA was obtained from a regional lymph node. In the absence of enlarged lymph nodes, FNA from primary lesions was carried out only if specifically requested by the patient and/or the treating physician.

\section{EUS technique}

A written informed consent was obtained from patientd before the procedure. Moderate sedation was given with the combination of midazolam and pentazocine.

EUS examinations were performed by experienced endoscopists (AA/MK/VS), who each had performed more than 2000 EUS examinations. All the cases were performed with the linear array echoendoscope (GF UCT 180, Olympus Medical Systems Corp, Tokyo, Japan) in the left lateral position. After evaluation of the esophagus and stomach, the echoendoscope was introduced in the first part of duodenum, and stabilized at the junction of the first and second parts of the duodenum. Portal vein and lower common bile were focused from the first part of the duodenum, after localization, and the common bile duct was traced towards the liver hilum by counterclockwise rotation and pull-back maneuver. Either the bile duct, intrahepatic radical or gallbladder body were traced towards the lesion, which usually has an echotexture different from the surrounding tissue ( $\triangleright$ Fig. $\mathbf{1 a}$ and $\triangleright$ Fig. 1b). Tumor was evaluated for involvement of hepatic artery or portal vein, liver metastasis or lymph node enlargement. Vascular involvement by tumor was considered to be present if any of the following were noted: loss of the hyperechoic interface between tumor and vessel, tumor thrombus within vessel lumen, or vessel encasement. Doppler examination was performed prior to FNA to rule out any intervening vessel. FNA was obtained from the primary mass, liver lesions, or from the metastatic appearing node ( $\boldsymbol{F} \mathbf{F i g . 1 c}$ - Fig. 1d, - Fig. 1e). In case of sampling of gallbladder lesions, direct puncture of the mass was done, and puncturing of normal wall, and the transluminal passage was avoided. A 22 G needle was used for sampling (Echotip Ultra, Cook Ireland Ltd, Limerick, Ireland, or Expect Slimline, Boston Scientific Corp, Massachusetts, United States), and a stylet was not used. At least four passes were made during each procedure and the needle was moved eight to 10 times during each pass. Suction was not used routinely, unless the first pass had insufficient material. After aspiration, material was expressed on slides and both alcohol-fixed and air-dried slides were prepared. Rapid onsite cytology examination (ROSE) was not performed routinely. A final cytological diagnosis was made by an experienced team of cytopathologists (KV/PB). Confirmation of metastasis to the lymph node or liver was considered sufficient for diagnosis of malignancy, irrespective of whether the primary tumor was targeted or FNA was negative from the primary tumor site. In the absence of cholangitis, routine antibiotics were not used. 

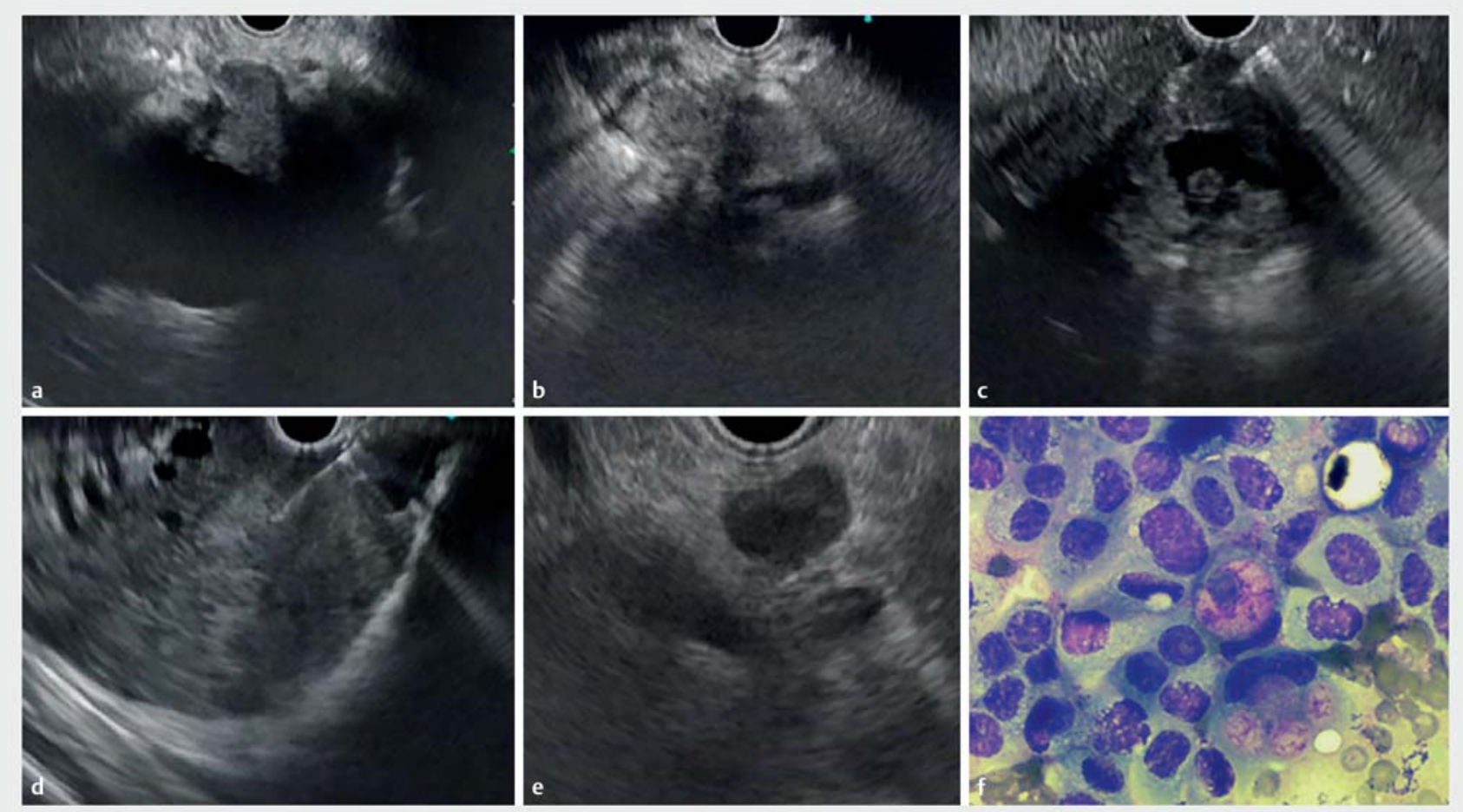

- Fig. 1 a Well-defined mass at gallbladder neck. b Gallbladder mass with ill-defined margins. $\mathbf{c}$ FNA from gallbladder mass. $\mathbf{d}$ FNA from liver lesion. e Enlarged pericholedochal node. $\mathbf{f}$ Cytology of gallbladder mass showing adenocarcinoma.

Patients were advised to visit the emergency room or contact the primary physician in case of development of new-onset or worsening of pain, fever or gastrointestinal bleed.

\section{Final diagnostic criteria and outcome parameters}

EUS-FNA diagnosis was categorized as malignant or non-malignant which was tested against the final diagnosis.

FNA reports were categorized as diagnostic of malignancy, ( $\triangleright$ Fig. 1f), suspicious for malignancy, atypical cytology, inconclusive or benign disease. For evaluation of EUS performance, specimens reported as diagnostic of malignancy were categorized as malignant and all other reports (suspicious/inconclusive/atypical or benign) were considered as non-malignant. For the lymph node FNA reports, reactive was considered as benign. If patients underwent EUS-FNA more than once, the final FNA report was considered for evaluation.

A final diagnosis of malignancy was made if there was definite evidence of malignancy on EUS-FNA sample, ERCP brushings (obtained from bile duct stricture site), percutaneous sample or surgical specimen. Clinical course compatible with malignancy was also considered as evidence of malignancy. Patients who were lost to follow-up were not included in the final analysis.

Data were reviewed for occurrence of new-onset pain, cholangitis, bleeding, or bile leak with peritonitis.

\section{Statistical analyses}

Categorical variables were expressed as frequencies and percentages and continuous variables as mean and standard deviation. Sensitivity, specificity, diagnostic accuracy, positive predictive value (PPV) and negative predictive value (NPV) were calculated. Sensitivity and specificity were calculated as the proportion of malignant and non-malignant FNA among all malignant and benign cases, respectively. PPV and NPV were calculated as proportion of true positive among all positive cases and proportion of true negative among all negative cases. SPSS software version 17.0 was used for analysis.

\section{Results}

Three hundred six patients underwent evaluation for proximal biliary obstruction and of them, 107 had biliary obstruction due to gallbladder mass lesion. FNA was not taken in six patients, as the lesion was resectable in five patients and because of intervening vessel in one patient.

One hundred one patients underwent FNA and their demographic and baseline characteristics are listed in $>$ Table 1. Ten lesions were resectable and of them, FNA was taken from a lymph node in six patients and the primary mass in four patients. Three patients had confirmation of diagnosis after second FNA and six patients had undergone ERCP previously with stent in situ. Mean size of the mass was $29.74 \pm 12.01 \mathrm{~mm}$.

FNA was carried out from one site in 83 patients and 18 patients had FNA from two sites. Fifty-eight had FNA only from 


\begin{tabular}{l|l|}
\hline Table 1 Demographics and baseline characteristics of 101 patients undergoing FNA. \\
\hline Age (mean \pm SD), years & $58.42 \pm 13.37$ \\
\hline Sex F:M & $73 F: 28 \mathrm{M}$ \\
\hline Bilirubin (mean \pm SD), mg/dL & $9.96 \pm 5.28$ \\
\hline Serum alkaline phosphatase (mean \pm SD), IU/L & $591.12 \pm 384.49$ \\
\hline Resectable lesions, $n(\%)$ & $10(9.90)$ \\
\hline Primary confluence blocked, $n(\%)$ & $81(80.19 \%)$ \\
\hline Number of patients requiring more than one FNA, n(\%) & $3(2.97 \%)$ \\
\hline Stent in situ, n(\%) & $6(5.94 \%)$ \\
\hline Size of the mass (mean \pm SD), mm & $29.74 \pm 12.01$ \\
\hline FNA, fine-needle aspiration & \\
\hline
\end{tabular}

the gallbladder, 23 and two patients underwent FNA only from lymph node or liver, respectively. Sixteen patients underwent FNA from both the gallbladder and lymph node, one from both lymph node and liver and one from both liver and gallbladder.

Of 101patients undergoing FNA, 89 were positive for malignancy from at least one of the sites. Of the remaining 12 lesions, four were suspicious, two inconclusive, and six were benign. Among 12 patients where FNA was non-malignant, three were finally diagnosed as having malignancy based on percutaneous FNA findings, three had malignancy on surgical specimen, three were diagnosed to be malignancy based on compatible clinical course, one lesion was benign on surgical specimen, and two were lost to follow-up. In the final analysis, 98 lesions were malignant and one lesion was benign. Sensitivity of the study was $90.81 \%$ (89/98), specificity was $100 \%(1 / 1)$, PPV was $100 \%(89 / 89)$, and NPV was $10 \%(1 / 10)$. The test was accurate in 90 patients (accuracy $90.90 \%$ ). Findings from EUS-FNA and final diagnoses are shown in $>$ Table 2 and the overall yield of EUS-FNA in relation to the final diagnosis is shown in $>$ Table 3.

\section{Adverse events}

Two patients developed self-limiting abdominal pain. There were no serious AEs like procedure-related cholangitis, bleeding, or bile leak in any patient.

\section{Discussion}

Despite widespread use of EUS-FNA in various pancreaticobiliary lesions, published data are scarce regarding its role in gallbladder mass lesions. The current study included the largest number of patients with gallbladder lesions, and specifically included patients with jaundice due to compression of the biliary system by the gallbladder mass lesion. About 265 to $34 \%$ of patients with gallbladder cancer have jaundice as the presenting complaint $[7,8]$. Jaundice is a marker of poor prognosis and most of such lesions are unresectable $[9,10]$, have limited survival $[11,12]$, and chemotherapy has limited benefit [13]. Although gallbladder mass lesions presenting with jaundice are mostly due to malignancy, alternate diseases with better prog- nosis, such as lymphoma, and benign diseases such as Mirrizzi syndrome, xanthogranulomatous cholecystitis, and tuberculosis need to be ruled out. In the current study, only one patient had a benign diagnosis, as patients with obvious benign lesions such as common bile duct stone (CBD) and Mirrizzi syndrome were excluded.

Jaundice in patients with gallbladder cancer is due to infiltration of the common hepatic or CBD. Because of close proximity of the CBD to the portal vein and hepatic artery, these vessels are simultaneously involved and curative surgery is not possible. In the current study, 86 of 101 patients (85.14\%) were found to have unresectable disease during EUS evaluation. In a large series of patients with gallbladder cancer and jaundice, only $7 \%$ underwent surgery with curative intent, $5 \%$ had negative margin, and median survival was only 6 months and none of the patients with jaundice survived beyond 2 years [8]. Because of extremely poor prognosis and limited treatment options and survival, pathological diagnosis is necessary to rule out potentially treatable alternate pathologies, and to counsel the patient and family. Available sampling techniques are ultrasound (USG) [22-25], and computed tomography (CT)-guided cytology/biopsy, ERCP brushing and biopsy and EUS-FNA/EUSfine needle biopsy (EUS-FNB). US-guided sampling is one of the most common modalities, with sensitivity more than $88 \%$ but it may be associated with bile leak and pain [22,24], and small mass lesions presenting with jaundice may not be visualized with it. ERCP brushings, though available, are associated with potential complications, hence the technique is reserved for patients with cholangitis or intractable pruritus [26, 27]. Moreover, yield of ERCP brushings is low [28], and after ERCP and stent placement, it may be difficult to visualize the mass, especially if it is small. EUS provides excellent resolution of the gallbladder from the antrum or duodenal bulb and even a small mass can be seen, and FNA can be obtained. The gallbladder also can be visualized from the proximal stomach, however, trans-gastric FNA is less favorable because of the intervening portal vein.

EUS is available in most endoscopy centers, and is becoming a preferred modality for evaluation of biliary obstruction. FNA can be done during the same procedure, obviating the need 
Table2 EUS-FNA for evaluation of gallbladder lesions in 101 patients.

\begin{tabular}{|c|c|c|}
\hline Site of FNA $(n=101)$ & FNA diagnosis & Final diagnosis \\
\hline \multirow[t]{4}{*}{ FNA from GB $(n=58)$} & Positive-51 & Malignant-51 \\
\hline & Suspicious-3 & $\begin{array}{l}\text { Malignant-3 (1 malignant on percutaneous FNA, } 1 \text { malignant on } \\
\text { surgical specimen; } 1 \text { compatible clinical course) }\end{array}$ \\
\hline & Inconclusive-2 & $\begin{array}{l}\text { Malignant-2 (1 malignant on surgical specimen; } 1 \text { compatible } \\
\text { clinical course) }\end{array}$ \\
\hline & Benign-2 & $\begin{array}{l}\text { Malignant-1 ( } 1 \text { malignant on surgical specimen) } \\
1 \text { lost to follow up }\end{array}$ \\
\hline \multirow[t]{2}{*}{ FNA from lymph node $(n=23)$} & Positive-20 & Malignant-20 \\
\hline & Reactive-3 & $\begin{array}{l}\text { Malignant-2 ( } 1 \text { malignant on percutaneous FNA, } 1 \text { compatible } \\
\text { clinical course) } \\
1 \text { lost to follow up }\end{array}$ \\
\hline FNA from liver lesion $(n=2)$ & Positive-2 & Malignant-2 \\
\hline \multirow[t]{5}{*}{ FNA from lymph node and GB $(n=16)$} & Both positive- 9 & Malignant-9 \\
\hline & (GB positive, $L N$ reactive)- 4 & Malignant-4 \\
\hline & (GB inconclusive, $L N$ positive)-1 & Malignant-1 \\
\hline & (GB suspicious, LN reactive)- 1 & Malignant-1 (Percutaneous FNA malignant) \\
\hline & (GB benign, $L N$ reactive)- 1 & Benign-1 (on surgical specimen) \\
\hline FNA from liver lesion and GB $(n=1)$ & Both Positive-1 & Malignant-1 \\
\hline \multirow[t]{2}{*}{ FNA from both liver and lymph node $(n=1)$} & Both Positive-1 & Malignant-1 \\
\hline & $\begin{array}{l}\text { Total FNA diagnosis: } \\
\text { Malignant- } 89 \\
\text { Non-malignant-12 }\end{array}$ & $\begin{array}{l}\text { Final diagnosis } \\
\text { Malignant- } 98, \\
\text { Lost to follow up-2 } \\
\text { Benign-1 }\end{array}$ \\
\hline
\end{tabular}

- Table 3 Overall yield of EUS-FNA.

\begin{tabular}{|l|l|}
\hline \multirow{2}{*}{ Final diagnosis, $n-99$} & Malignant $(n=98)$ \\
\hline & Benign $(n=1)$ \\
\hline
\end{tabular}

\begin{tabular}{|l|l|}
\hline \multicolumn{2}{|l|}{ EUS FNA diagnosis, n-99 } \\
\hline Malignant (89) & Non-malignant (10) \\
\hline 89 & 9 \\
\hline 0 & 1 \\
\hline
\end{tabular}

for a second procedure by a radiologist. As a departmental policy, in patients with gallbladder mass, we attempt pathological diagnosis first with EUS-FNA, and ERCP is performed only for palliation of itching or cholangitis. Because of concern about needle-track seeding, EUS-FNA is reserved specifically for patients with unresectable disease, and those who have resectable lesions with high suspicion of malignancy can be directly referred for surgery. Exceptions are suspicion of benign disease in young patients, presence of large nodes suggesting tuberculosis or lymphoma, or if pathological confirmation is requested by the surgeon or patient before the surgery. EUS-FNA of gallbladder mass lesions has been reported, however, all the previous studies had small sample size, and none of the studies specifically included patients with jaundice. Jacobson et al.
[19] first reported the role of EUS-FNA in gallbladder mass, FNA was positive in four of five proven malignant masses and negative in a single benign case. Hijoka et al. [18] reported that accuracy of EUS in differentiating gallbladder malignancy from xanthogranulomatous cholecystitis, accuracy of EUS-FNA for detecting malignancy and for the final diagnosis was $93.3 \%$ and $80 \%$ respectively. In a series of seven patients with proven gallbladder malignancy, Meara et al. [21] found malignancy in six patients. Varadarajulu et al. [20] performed EUS-FNA in five patients with malignancy and one benign case and FNA diagnosis was correct in all the cases.

- Table 4 highlights the diagnostic performance of the previous and current studies. The current study, with the largest data set available to date, confirms the high sensitivity of EUS- 
- Table 4 Studies comparing the role of EUS-FNA for gallbladder mass.

\begin{tabular}{|c|c|c|c|c|c|}
\hline Author & Year & No & Final diagnosis & Sensitivity & Specificity \\
\hline Jacobson et al. [19] & 2003 & 6 & $\begin{array}{l}\text { Benign } 1 \\
\text { Malignant } 5\end{array}$ & $80 \%$ & $100 \%$ \\
\hline Varadarajulu et al. [20] & 2005 & 6 & $\begin{array}{l}\text { Malignant } 5 \\
\text { Benign } 1\end{array}$ & $100 \%$ & $100 \%$ \\
\hline Meara Et al [21] ${ }^{1}$ & 2007 & 7 & Malignant 7 & $85.71 \%$ & - \\
\hline Hijioka et al. [18] & 2010 & 15 & $\begin{array}{l}\text { Malignant } 10 \\
\text { Benign } 5\end{array}$ & $90 \%$ & $100 \%$ \\
\hline Present study & 2018 & 101 & $\begin{array}{l}\text { Malignant: } 98 \\
\text { Benign } 1\end{array}$ & $90.81 \%$ & $100 \%$ \\
\hline
\end{tabular}

FNA for gallbladder cancer lesions with biliary obstruction. NPV was low, hence lesions with negative results should undergo further evaluation. EUS-FNA appeared safe, none of the patients had serious AEs in the form of bile leak, cholangitis or bleed. This may be due to close contact of the gallbladder with the duodenum, without any need to going through the gallbladder lumen.

The strength of the current study is the large and uniform population of patients with obstructive jaundice due to gallbladder mass lesions, mostly unresectable, where pathological confirmation is mandatory. The limitation of the study is that rapid onsite evaluation was unavailable, which could have further increased accuracy. Newer EUS-FNB needles have now become available for tissue diagnosis, which may further improve accuracy of EUS FNA [29]. In the current study, EUS was not compared with other modalities for resectability, hence we cannot comment on its accuracy for evaluation of resectability. Because of the retrospective design, risk of missing information, especially about adverse events, cannot be ignored. Although we tried to differentiate gallbladder and bile duct lesions based on predominant site of involvement, overlap between these lesions cannot be ignored, and there may be inadvertent inclusion of cholangiocarcinoma in our cohort.

\section{Conclusion}

To conclude, we found EUS-FNA to be a sensitive modality for evaluation of gallbladder mass lesions with biliary obstruction. However the low NPV suggests that negative FNA does not rule out malignancy, and those lesions should be further investigated.

\section{Acknowledgements}

The authors thank Meetu Sharma for help in data compilation.

\section{Competing interests}

None

References

[1] Nanda Kumar A, Gupta PC, Gangadharan P et al. Geographic pathology revisited: Development of an atlas of cancer in India. Int J Cancer 2005; 116: $740-754$

[2] Batra Y, Pal S, Dutta U et al. Gallbladder cancer in India: a dismal picture. J Gastroenterol Hepatol 2005; 20: 309-314

[3] Lazcano-Ponce EC, Miquel JF, Muñoz N et al. Epidemiology and molecular pathology of gallbladder cancer. CA Cancer J Clin 2001; 51: $349-364$

[4] Ito H, Matros E, Brooks DC et al. Treatment outcomes associated with surgery for gallbladder cancer: a 20-year experience. J Gastrointest Surg 2004; 8: $183-190$

[5] Cubertafond P, Gainant A, Cucchiaro G. Surgical treatment of 724 carcinomas of the gallbladder. Results of the French Surgical Association Survey. Ann Surg 1994; 219: 275-280

[6] Fong Y, Jarnagin W, Blumgart LH. Gallbladder cancer: comparison of patients presenting initially for definitive operation with those presenting after prior non curative intervention. Ann Surg 2000; 232: 557-669

[7] Yang XW, Yuan JM, Chen JY et al. The prognostic importance of jaundice in surgical resection with curative intent for gallbladder cancer. BMC Cancer 2014; 14: 652

[8] Hawkins WG, DeMatteo RP, Jarnagin WR et al. Jaundice predicts advanced disease and early mortality in patients with gallbladder cancer. Ann Surg Oncol 2004; 11: 310-315

[9] Kumaran V, Gulati S, Paul B et al. The role of dual-phase helical CT in assessing resectability of carcinoma of the gallbladder. Eur Radiol 2002; 12: $1993-1999$

[10] Bismuth H, Castaing D, Traynor O. Resection or palliation: priority of surgery in the treatment of hilar cancer. World J Surg 1988; 12: 39 47

[11] Regimbeau JM, Fuks D, Bachellier P et al. Prognostic value of jaundice in patients with gallbladder cancer by the AFC- gallbladder C-2009 study group. Eur J Surg Oncol 2011; 37: 505-512

[12] Misra S, Chaturvedi A, Misra NC et al. Carcinoma of the gallbladder. Lancet Oncol 2003; 4: 167-176 
[13] Fareed MM, DeMora L, Esnaola NF et al. Concurrent chemoradiation for resected gallbladder cancers and cholangiocarcinomas. J Gastrointest Oncol 2018; 9: $762-768$

[14] Fritscher-Ravens A, Broering DC, Sriram PV et al. EUS-guided fineneedle aspiration cytodiagnosis of hilar cholangiocarcinoma: a case series. Gastrointest Endosc 2000; 52: 534-540

[15] Fritscher-Ravens A, Broering DC, Knoefel WT et al. EUS-guided fineneedle aspiration of suspected hilar cholangiocarcinoma in potentially operable patients with negative brush cytology. Am J Gastroenterol 2004; 99: 45 - 51

[16] Eloubeidi MA, Chen VK, Jhala NC et al. Endoscopic ultrasound-guided fine needle aspiration biopsy of suspected cholangiocarcinoma. Clin Gastroenterol Hepatol 2004; 2: 209-213

[17] DeWitt J, Misra VL, Leblanc JK et al. EUS-guided FNA of proximal biliary strictures after negative ERCP brush cytology results. Gastrointest Endosc 2006; 64: 325 - 333

[18] Hijioka S, Mekky MA, Bhatia V et al. Can EUS-guided FNA distinguish between gallbladder cancer and xanthogranulomatous cholecystitis? Gastrointest Endosc 2010; 72: 622 - 627

[19] Jacobson B, Pitman M, Brugge W. EUS-guided FNA for the diagnosis of gallbladder masses. Gastrointest Endosc 2003; 57: 251-254

[20] Varadarajulu S, Eloubeidi M. Endoscopic ultrasound-guided fine-needle aspiration in the evaluation of gallbladder masses. Endoscopy 2005; 37: $751-754$

[21] Meara RS, Jhala D, Eloubeidi MA et al. Endoscopic ultrasound-guided FNA biopsy of bile duct and gallbladder: analysis of 53 cases. Cytopathology 2006; 17: $42-9$
[22] Zargar SA, Khuroo MS, Mahajan R et al. US-guided fine-needle aspiration biopsy of gallbladder masses. Radiology 1991; 179: 275-278

[23] Kumar R, Srinivasan R, Gupta N et al. Spectrum of gallbladder malignancies on fine-needle aspiration cytology: 5 years retrospective single institutional study with emphasis on uncommon variants. Diagn Cytopathol 2017; 45: $36-42$

[24] Rana C, Krishnani N, Kumari N. Ultrasound-guided fine needle aspiration cytology of gallbladder lesions: a study of 596 cases. Cytopathology 2016; 27: 398-406

[25] Pandey M, Sood BP, Shukla RC et al. Carcinoma of the gallbladder: role of sonography in diagnosis and staging. J Clin Ultrasound 2000; 28: $227-232$

[26] Saluja SS, Gulati M, Garg PK et al. Endoscopic or percutaneous biliary drainage for gallbladder cancer: a randomized trial and quality of life assessment. Clin Gastroenterol Hepatol 2008; 6: 944-950

[27] Miura S, Kanno A, Masamune A et al. Risk factors for recurrent biliary obstruction following placement of self-expandable metallic stents in patients with malignant perihilar biliary stricture. Endoscopy 2016; 48: $536-545$

[28] Singh A, Gelrud A, Agarwal B. Biliary strictures: diagnostic considerations and approach. Gastroenterology report 2014; 3: 22 - 31

[29] Khan MA, Grimm IS, Ali B et al. A meta-analysis of endoscopic ultrasound-fine-needle aspiration compared to endoscopic ultrasoundfine-needle biopsy: diagnostic yield and the value of onsite cytopathological assessment. Endosc Int Open 2017; 5: E363-E375 